\title{
Effects of Rivastigmine in Alzheimer's Disease Patients with and Without Hallucinations
}

\author{
Jeffrey Cummings ${ }^{\mathrm{a}, *}$, Murat Emre ${ }^{\mathrm{b}}$, Dag Aarsland ${ }^{\mathrm{c}}$, Sibel Tekin $^{\mathrm{d}}$, Nalina Dronamraju ${ }^{\mathrm{d}}$ and Roger Lane ${ }^{\mathrm{d}}$ \\ ${ }^{a}$ Mary S. Easton Center for Alzheimer's Disease Research at UCLA, Los Angeles, CA, USA \\ ${ }^{\mathrm{b}}$ Department of Neurology, Behavioural Neurology and Movement Disorders Unit, Istanbul Faculty of Medicine, \\ Istanbul University, Istanbul, Turkey \\ ${ }^{\mathrm{c}}$ Norwegian Centre for Movement Disorders, Psychiatric Clinic, Stavanger University Hospital, Stavanger, Norway \\ ${ }^{\mathrm{d}}$ Novartis Pharmaceuticals Corporation, East Hanover, NJ, USA
}

Handling Associate Editor: Sandrine Andrieu

Accepted 20 December 2009

\begin{abstract}
Hallucinations in Alzheimer's disease (AD) may indicate greater cortical cholinergic deficits. Rivastigmine has shown larger treatment benefits versus placebo in dementia with Lewy bodies and Parkinson's disease dementia patients with hallucinations. In this retrospective, hypothesis-generating analysis, we investigated whether hallucinations in AD were associated with greater treatment benefits with rivastigmine. Data were pooled from two randomized, double-blind, 6-month, mild-tomoderate AD trials comparing rivastigmine with placebo. Co-primary efficacy parameters were the Alzheimer Disease Assessment Scale-cognitive subscale (ADAS-cog) and Clinician's Interview-Based Impression of Change plus Caregiver Input (CIBIC-plus). Efficacy data were analyzed for two sub-populations: those with and those without hallucinations at baseline. Of 927 patients, $194(21 \%)$ reported hallucinations at baseline. Hallucinators tended to have greater decline on placebo on all outcome measures. On the ADAS-cog, mean rivastigmine - placebo differences of 3.7 points in hallucinators and 2.2 points in non-hallucinators were reported at 6 months (both $p<0.001$ ). In hallucinators, a significant rivastigmine - placebo difference of -1.0 points (a beneficial effect) was seen on the CIBIC-plus at 6 months $(p<0.001)$. Non-hallucinators showed a smaller significant treatment difference of -0.3 points $(p<0.05)$. Interaction testing suggested that differences in treatment effects were significant between hallucinators and non-hallucinators. Hallucinations predicted greater treatment responses to oral rivastigmine.
\end{abstract}

Keywords: Alzheimer's disease, cholinesterase inhibitor, hallucinations, rivastigmine

\section{INTRODUCTION}

Alzheimer's disease (AD) is the most common cause of dementia, 4.6 million new cases of which arise each year worldwide [1]. In addition to amyloid deposits and neurofibrillary tangles, AD is characterized by deficits

*Correspondence to: Professor Jeffrey Cummings, Mary S. Easton Center for Alzheimer's Disease Research,10911 Weyburn Ave; Suite 200, Los Angeles, CA 90095-7226, USA. Tel.: +1 310794 3365; Fax: +1 310206 5287, E-mail: jcummings@mednet.ucla.edu. in cortical cholinergic innervation, caused by loss of cortical cholinergic neurons in the basal forebrain with a consequent reduction in choline acetyltransferase activity [2]. This cholinergic deficit contributes to the cognitive dysfunction seen in AD.

In patients with Lewy body pathology, greater cortical cholinergic deficits have been reported in hallucinators versus non-hallucinators [3]. Data also support the proposal that behavioral and psychological symptoms (such as hallucinations) in $\mathrm{AD}$ are related, in part, to cholinergic neuronal loss and a subsequent decline in 
acetylcholine levels in regions of the brain responsible for behavioral and emotional responses [4]. Hallucinations are common in $\mathrm{AD}$, present in $20-40 \%$ of patients [5-8], with visual hallucinations the most common form $[9,10]$.

The proposed association of hallucinations with cortical cholinergic deficits, and previous findings in dementia with Lewy bodies (DLB) and dementia associated with Parkinson's disease (PDD) patients with and without hallucinations [11,12], provided a rationale to investigate the effects of rivastigmine in AD patients with and without hallucinations. The aim of this study was to perform a pooled analysis of two similar large randomized clinical trials in mild-tomoderate AD that were performed as part of the ADENA (Alzheimer's Disease Treatment with ENA-713) program, in order to investigate the effects of rivastigmine versus placebo in the sub-populations of patients with and without hallucinations at baseline.

\section{METHODS}

\section{Patients}

This was a pooled analysis of two large randomized placebo-controlled studies of rivastigmine capsules in AD $[13,14]$. Patients enrolled in either of the two studies were required to fulfill the criteria for dementia of the Alzheimer's type as described in the fourth edition of the Diagnostic and Statistical Manual of Mental Disorders [15], have probable AD according to the criteria of the National Institute of Neurological and Communicative Disorders and Stroke and the Alzheimer's Disease and Related Disorders Association [16], and score between 10 and 26 on the Mini-Mental State Examination (MMSE). Age ranges varied between 45 and 89 in one study [13], and between 50 and 85 in the other [14]. All patients had a responsible caregiver, and both patients and caregivers provided written, informed consent. Patients with concomitant diseases (apart from causes of dementia other than AD, or severe and unstable medical illnesses such as serious cardiac disease, severe obstructive pulmonary disease, or aggressive malignancies) were included. Patients were permitted to continue with most medications for coexistent diseases. Anti-cholinergic drugs, health food supplements containing acetylcholine precursors, putative memory enhancers, and insulin were not permitted. The use of psychotropic drugs was prohibited, apart from small doses of short acting benzodiazepines, chloral hydrate, or haloperidol in one trial [19], and occasional use of chloral hydrate in the other [13]. Procedures in both studies were in accordance with the Helsinki Declaration as revised in 1983 [13,14].

\section{Study design}

This analysis examined pooled patient data from two multicenter, randomized, double-blind, placebocontrolled, 3-arm, parallel-group 6-month trials of rivastigmine capsules in de novo patients with mildto-moderate AD $[13,14]$. These studies were chosen due to their almost identical study design, with flexible dosing up to $12 \mathrm{mg} /$ day in two divided daily doses, which facilitated pooling of the data. The other trials of rivastigmine in $\mathrm{AD}$ included in the rivastigmine registration submission package were excluded from this analysis as they enforced a fixed dose of rivastigmine or employed three-times daily dosing [17-19]. These variations in study design could confound the analysis of pooled data.

Both studies consisted of an initial fixed dose titration phase of between 7 and 12 weeks, in which dosages were increased weekly and decreases in doses due to adverse events were not permitted. During the dose maintenance phase, the maximum tolerated amount was maintained, and decreases of doses due to side effects were possible within an assigned range. Patients were randomized to $1-4 \mathrm{mg} /$ day or 6-12 $\mathrm{mg}$ /day target dose rivastigmine, or placebo; though for the purposes of this pooled analysis only the higher target dose and placebo groups were included.

Co-primary efficacy outcome measures in both studies were the Alzheimer Disease Assessment Scalecognitive subscale (ADAS-cog) [20], to assess cognition, and the Clinician's Interview-Based Impression of Change plus Caregiver Input (CIBIC-plus) [21], to assess global performance. The Progressive Deterioration Scale (PDS) [22] was a secondary outcome measure, assessing ability to perform daily activities. Efficacy evaluations were performed at baseline and at Weeks 12, 18, and 26, or at withdrawal in the event of early termination. The Global Deterioration Scale (GDS) [23] and MMSE [24] were used as staging measures at baseline and Week 26. Neuropsychiatric symptoms were collected using the items of the Behavioral Pathology in Alzheimer's Disease Rating Scale (BEHAVE-AD) [25], used as an integrated aspect of the CIBIC-plus in this study. The BEHAVE-AD is a 25 -item scale covering the domains of paranoid and delusional ideation ( 7 items), hallucinations (5 items), 
activity disturbances (3 items), aggressiveness (3 items), diurnal rhythm disturbances (1 item), affective disturbance ( 2 items), and anxieties and phobias (4 items). Items are rated on a four-point scale $(0-$ 3) based on a clinical interview with a caregiver, with scores assigned according to the severity of symptoms observed. A score of zero in a specific item indicates absence of a symptom and a score of three represents the most severe category.

The current additional analyses were performed in the AD patient population based on the presence of hallucinations (including both visual and non-visual hallucinations) at baseline. The presence or absence of any hallucinations was recorded using the items of the BEHAVE-AD. Efficacy data were analyzed for two sub-populations: those with and those without hallucinations at baseline. All patients who received at least one dose of study medication and who had a subsequent safety evaluation were included in the safety population. Efficacy populations were: classical intention to treat (ITT), modified ITT (mITT), and traditional observed cases (OC). The ITT population included all randomized patients, whether or not they received treatment. In the case of missing assessments, a retrieved drop-out assessment was used; if there was no retrieved drop-out assessment, the last prior observation available was carried forward as an imputed value. The mITT population comprised randomized patients with at least one evaluation while being treated for whom the immediately preceding assessment was imputed for subsequently missing evaluations or data. The OC population comprised all randomized patients with evaluations made while on study drug at all of the designated assessment times. No imputations were used for the OC analyses. The primary confirmatory analysis was based on the change from baseline at Week 26 in the ITT population.

\section{Statistical methods}

Within the two sub-populations (hallucinators and non-hallucinators), treatment differences on the ADAScog, PDS, and CIBIC-plus (as continuous scores) were compared between rivastigmine- and placebo-treated patients using analysis of covariance (ANCOVA), with treatment and country as factors and baseline scores as covariates. Treatment differences on the CIBICplus and BEHAVE-AD were compared using the van Elteren test, blocking for country. The prevalence of adverse events was compared between rivastigmineand placebo-treated patients using Fisher's Exact Test, and differences in assessments of vital signs and ECG evaluations between the two sub-populations were tested for statistical significance using one-way ANCOVA.

When assessing the results of the ADAS-cog, PDS, MMSE, and GDS, the validity and statistical significance of the subgroup analyses were evaluated by testing for interaction. This was done using an ANCOVA model with treatment, country and baseline hallucination status as factors with interaction between treatment and baseline hallucination status, and appropriate baseline score (ADAS-cog, PDS, etc.) as covariates. For the results of the CIBIC-plus and BEHAVE$\mathrm{AD}$, interaction tests were carried out with an analysis of variance (ANOVA) model, using treatment, country, and baseline hallucination status as factors with interaction between treatment and baseline hallucination status. This was an exploratory hypothesis-generating analysis with no correction for multiple comparisons.

\section{RESULTS}

Patients

A total of 1424 patients entered the two AD trials [18, 19]. At baseline, these patients had a mean age of 73.2 years, a mean duration of dementia of 39.1 months, and a mean baseline MMSE score of 19.8. The majority (61\%) were women. There were no differences of note between the baseline characteristics and demographics of the two study populations $[13,14]$. The groups included in this pooled analysis (6-12 mg/day target dose rivastigmine and placebo) comprised a total of 948 patients. Of these 948 patients, 463 patients on rivastigmine $6-12 \mathrm{mg} /$ day and 464 patients on placebo provided BEHAVE-AD data. Figure 1 illustrates the pooled patient flow through the trials. The baseline characteristics of patients who provided BEHAVE-AD data are shown in Table 1. There were no notable differences in baseline characteristics between these 927 patients and the total population of 1424 patients $[13,14]$. Hallucinations were present at baseline in $106(23 \%)$ and $88(19 \%)$ of patients on rivastigmine and placebo, respectively.

Of the individual items on the BEHAVE-AD, the paranoid and delusional ideation item was present at baseline in $69 \%$ of hallucinators and $37 \%$ of nonhallucinators; the diurnal rhythm disturbances item was present in $31 \%$ of hallucinators and $17 \%$ of nonhallucinators; and the aggressiveness item was present in $62 \%$ and $41 \%$ of hallucinators and non-hallucinators, 
Table 1

Baseline characteristics of $\mathrm{AD}$ patients in two large randomized clinical trials of rivastigmine who provided BEHAVE-AD data to confirm the presence or absence of hallucinations (safety population) $[13,14]$

\begin{tabular}{lccccc}
\hline Characteristic & \multicolumn{2}{c}{ Hallucinations at baseline } & & \multicolumn{2}{c}{ No hallucinations at baseline } \\
\cline { 2 - 3 } \cline { 5 - 6 } & Rivastigmine & Placebo & & Rivastigmine & Placebo \\
\hline$n$ & 106 & 88 & & 356 & 376 \\
Mean age (years) & 73.5 & 74.0 & & 72.3 & 73.5 \\
Men women (\%) & $33: 67$ & $33: 67$ & & $37: 63$ & $43: 57$ \\
Mean weight (kg) & 65.7 & 64.4 & & 66.6 & 66.8 \\
Caucasian (\%) & 99 & 95 & & 97 & 96 \\
Mean duration of AD (months) & 42.0 & 40.3 & & 37.7 & 39.5 \\
Mean baseline MMSE & 18.4 & 19.1 & & 20.3 & 20.2 \\
Mean baseline GDS* & 4.3 & 4.1 & & 3.9 & 4.0 \\
Mean baseline ADAS-cog* & 27.1 & 26.3 & & 21.8 & 21.6 \\
Mean baseline PDS* & 43.2 & 45.2 & & 56.6 & 56.4 \\
\hline
\end{tabular}

*Baseline values from ITT population; patient numbers may vary slightly from safety population.

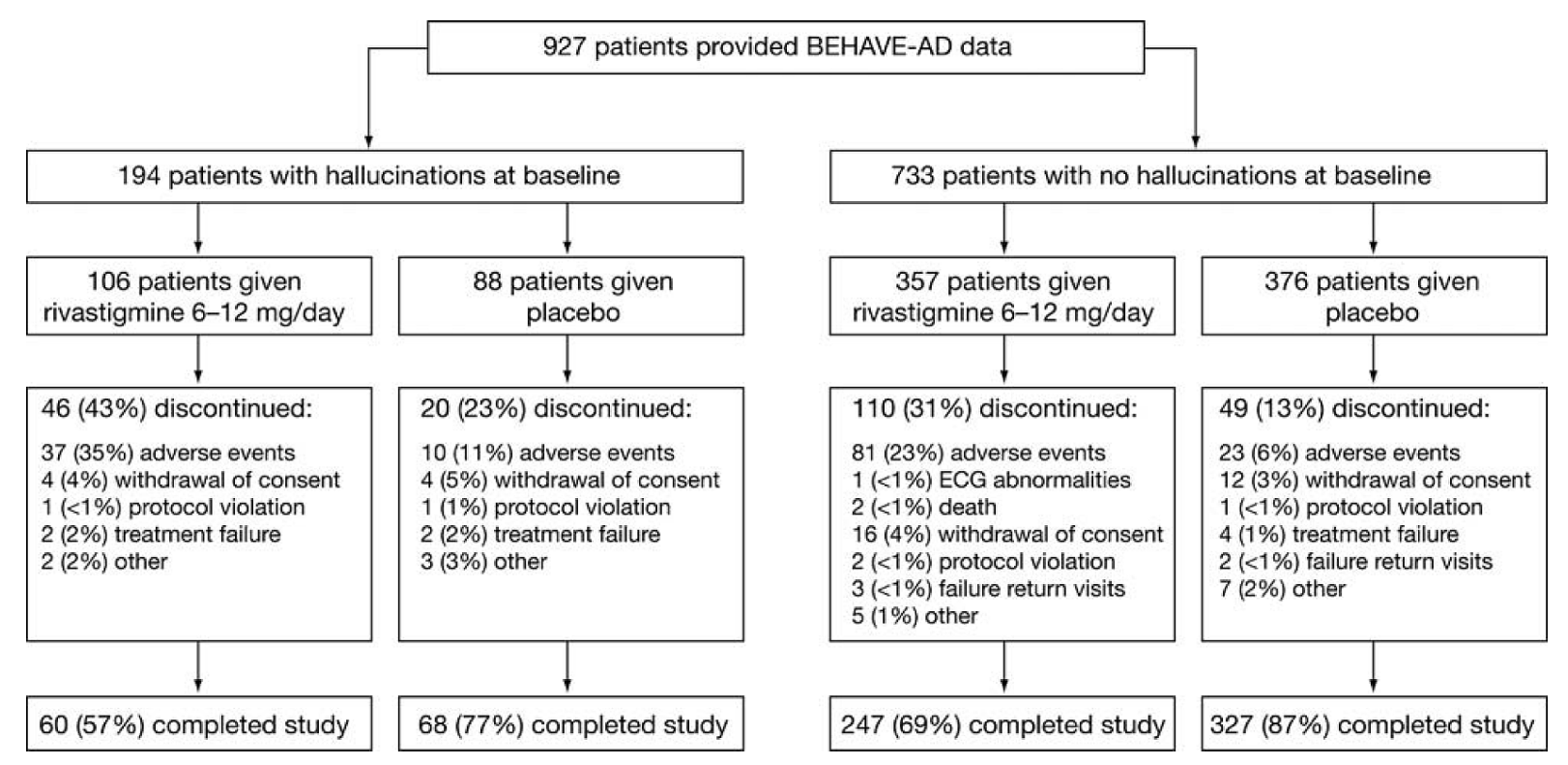

Fig. 1. Patient flow.

respectively. The activity disturbance item was reported at baseline for $82 \%$ of patients with and $59 \%$ of patients without hallucinations; the affective disturbances item was present in $59 \%$ of hallucinators and $44 \%$ of non-hallucinators; and the anxieties and phobias item was recorded at baseline for $84 \%$ of patients with hallucinations and $69 \%$ of patients without.

\section{Changes in the placebo group}

Patients with hallucinations at baseline tended to show a greater decline on placebo compared with patients without hallucinations, on all outcome measures (Table 2).
These findings were supported by the additional analyses performed on the mITT and OC populations. However, in the case of the $\mathrm{OC}$ analysis on the BEHAVE-AD, the trend for greater placebo decline in patients with hallucinations at baseline compared to those without was reversed, with non-hallucinators experiencing a slightly greater decline on placebo than hallucinators (a decline of 0.2 points compared with 0.1 points, respectively).

Doses

Over 6 months, the mean doses of rivastigmine in $\mathrm{mg} /$ day $( \pm \mathrm{SD})$ received by hallucinators and non- 


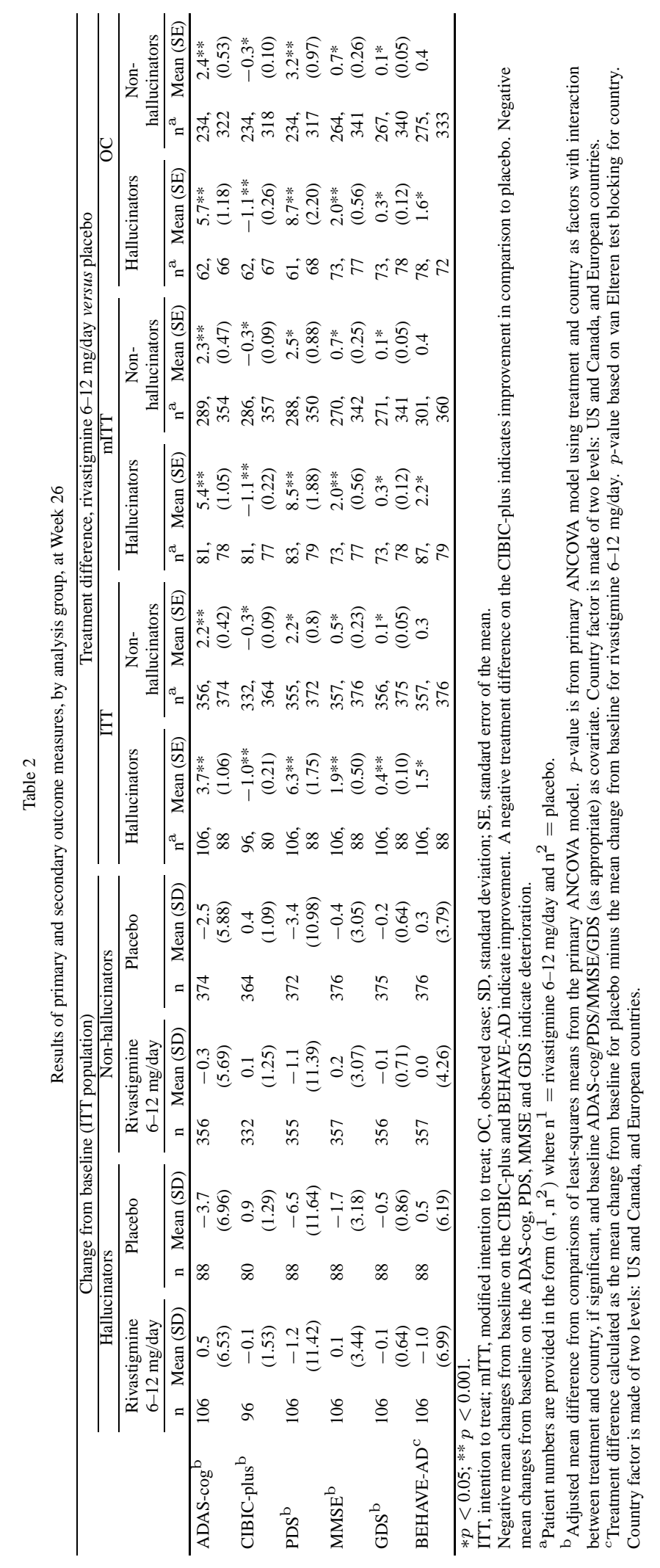


a) Hallucinations at baseline

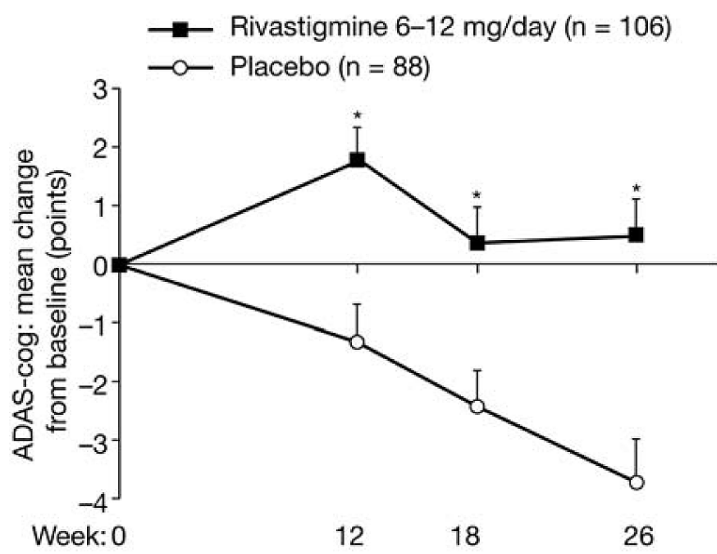

b) No hallucinations at baseline

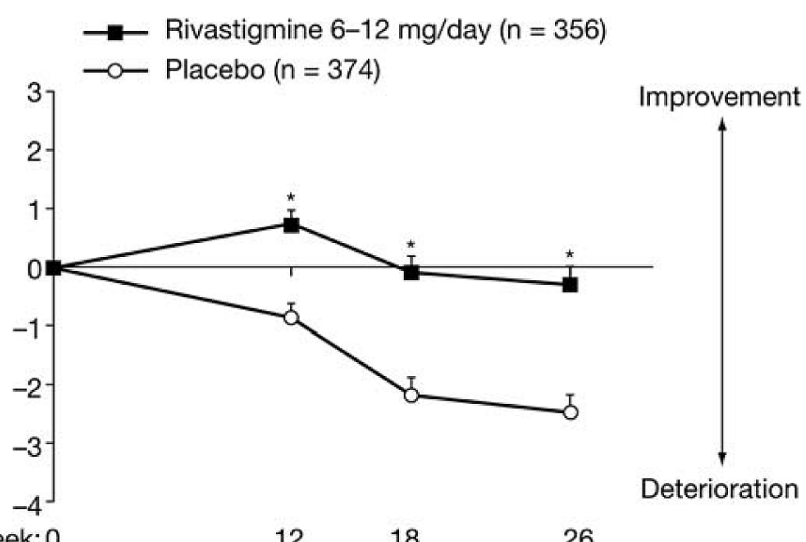

${ }^{*} p<0.05$

ITT analysis, $p$-value based on ANCOVA. Error bars indicate SEM.

Fig. 2. ADAS-cog scores in AD patients a) with and b) without hallucinations at baseline receiving either rivastigmine 6-12 mg/day or placebo (SEM, standard error of the mean).

hallucinators were $7.66 \pm 2.25$ and $7.91 \pm 2.32$ respectively. In the hallucinators group, $45 \%$ of patients on rivastigmine received the drug for the full 26 weeks, compared with $63 \%$ of patients randomized to placebo. In the group of patients without hallucinations at baseline, $55 \%$ of patients on rivastigmine received the drug for 26 weeks, compared to $71 \%$ of patients given placebo.

\section{Treatment responses}

\section{Cognitive performance}

At 6 months, a mean improvement of 0.5 points on the ADAS-cog was seen in patients with hallucinations at baseline treated with rivastigmine, while patients without hallucinations at baseline showed a 0.3point decline (Fig. 2). Significant rivastigmine - placebo treatment differences were seen in both populations (Table 2). These results were supported by the additional analyses performed on the mITT and OC populations. A statistically significant interaction between subgroup and treatment was observed for the mITT and OC populations (both $p<0.05$ ), indicating a differential treatment effect in the hallucinator and nonhallucinator groups.

\section{CIBIC-plus}

A significant rivastigmine - placebo difference was seen on the CIBIC-plus in hallucinators at 6 months (Table 2), with $36 \%$ versus $10 \%$ of patients improving and $37 \%$ versus $63 \%$ worsening, with rivastigmine and placebo respectively. Non-hallucinators showed a smaller, but still statistically significant treatment difference (Table 2), with $27 \%$ versus $19 \%$ of patients improving and $34 \%$ versus $43 \%$ worsening with rivastigmine and placebo, respectively. These results were supported by the mITT and OC analyses. Statistically significant interactions between baseline hallucination status and treatment were observed for the ITT, mITT and OC populations (all $p<0.05$ ), indicating significant differences in treatment effects between hallucinators and non-hallucinators.

\section{Activities of daily living}

Both hallucinators and non-hallucinators treated with rivastigmine showed a similar, slight decline (1.2 and 1.1 points, respectively) on the PDS at 6 months (Fig. 3). Significant rivastigmine - placebo differences were seen in both groups (Table 2). This treatment effect was statistically significantly different between hallucinators and non-hallucinators, as interactions between baseline hallucination status and treatment were significant for the ITT, mITT and OC populations (all $p<0.05)$.

\section{Neuropsychiatric symptoms}

As in previous studies analyzing overall and individual domain scores on the Neuropsychiatric Inventory $[31,32]$, both total BEHAVE-AD score and individual item scores were considered. Patients with hallucinations at baseline showed a significant rivastigmine - placebo difference on the BEHAVE-AD total 
a) Hallucinations at baseline

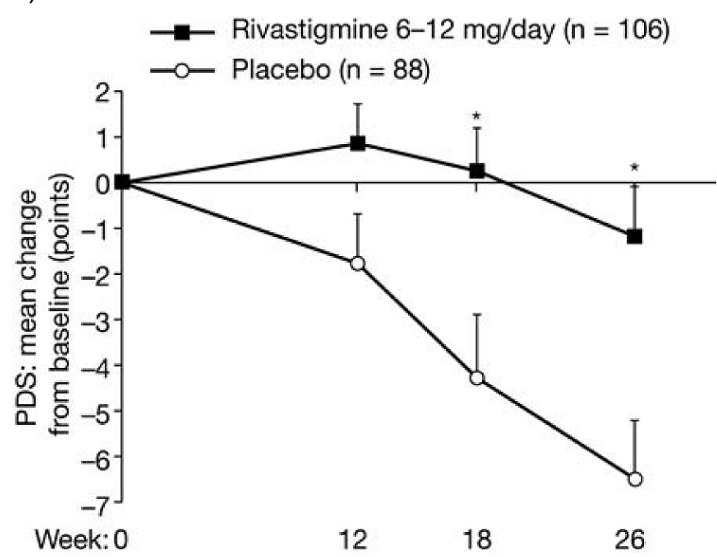

b) No hallucinations at baseline

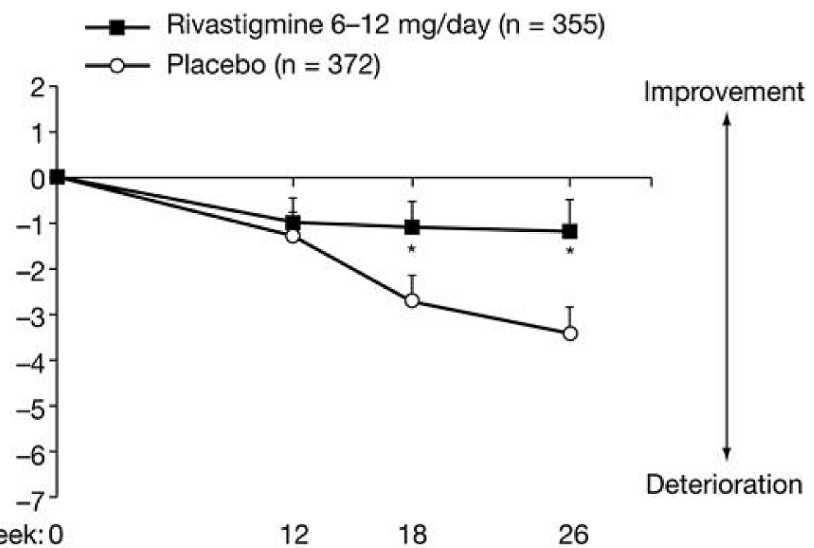

${ }^{*} p<0.05$

ITT analysis, $p$-value based on ANCOVA. Error bars indicate SEM.

Fig. 3. PDS scores in AD patients a) with and b) without hallucinations at baseline receiving either rivastigmine 6-12 mg/day or placebo (SEM, standard error of the mean).

score at 6 months (Table 2). These patients showed a non-significant treatment difference of 0.2 points on the hallucinations item at 6 months $(p=0.486)$; significant differences were seen on individual item scores for paranoid and delusional ideation and activity disturbance (all $p<0.05$ ) (Fig. 4). Non - hallucinators showed a non-significant treatment difference on the total BEHAVE-AD score (Table 2). There were significant differences on individual item scores for paranoid and delusional ideation and activity disturbance (all $p<0.05$ ) (Fig. 4). Rivastigmine - placebo differences on the BEHAVE-AD total score were not significantly different between hallucinators and non-hallucinators $(p=0.227)$.

\section{Staging measures}

At Week 26 all patients receiving rivastigmine showed significant treatment differences versus placebo on both the MMSE and GDS (Table 2). These results were supported by the additional analyses performed on the mITT and OC populations. Interactions between baseline hallucination status and treatment were found to be statistically significant in the ITT population only, for both the MMSE and GDS (both $p<0.05$ ).

\section{Safety and tolerability}

The most commonly reported adverse events for patients given rivastigmine, in both the hallucinator and non-hallucinator groups, were nausea and vomiting (Table 3), which in both groups occurred significantly more frequently with rivastigmine than with placebo $(p<0.05)$. These side-effects led to study discontinuation in $13 \%$ and $9 \%$ of hallucinators, and $12 \%$ and $6 \%$ of non-hallucinators, for nausea and vomiting respectively. Other frequently reported adverse events are listed in Table 3.

\section{Vital signs and ECG evaluation}

Patients without hallucinations at baseline treated with rivastigmine experienced a mean decrease in resting pulse rate over 6 months that was statistically significant compared to patients given placebo $(-2.3 \mathrm{bpm}$ versus $0.4 \mathrm{bpm}$ respectively, $p=0.001)$. There was no similar significant difference observed in patients with hallucinations at baseline $(-1.5 \mathrm{bpm}$ versus $-1.3 \mathrm{bpm}$ for rivastigmine and placebo respectively, $p=0.902$ ). This observation was reinforced by measurements of heart rate performed during ECG evaluations, where non-hallucinators, but not hallucinators, given rivastigmine experienced a statistically significant decrease over 6 months compared to those on placebo $(-2.2 \mathrm{bpm}$ versus $0.0 \mathrm{bpm}, p=0.019$ for non-hallucinators; -0.2 bpm versus $-1.1 \mathrm{bpm}, p=0.659$ for hallucinators). There were no other significant differences observed between patients randomized to rivastigmine and those given placebo.

\section{Adjustment for multiple comparisons}

The results reported here are intended for hypothesis generation only, and no correction for multiplicity 
a) Hallucinations at baseline

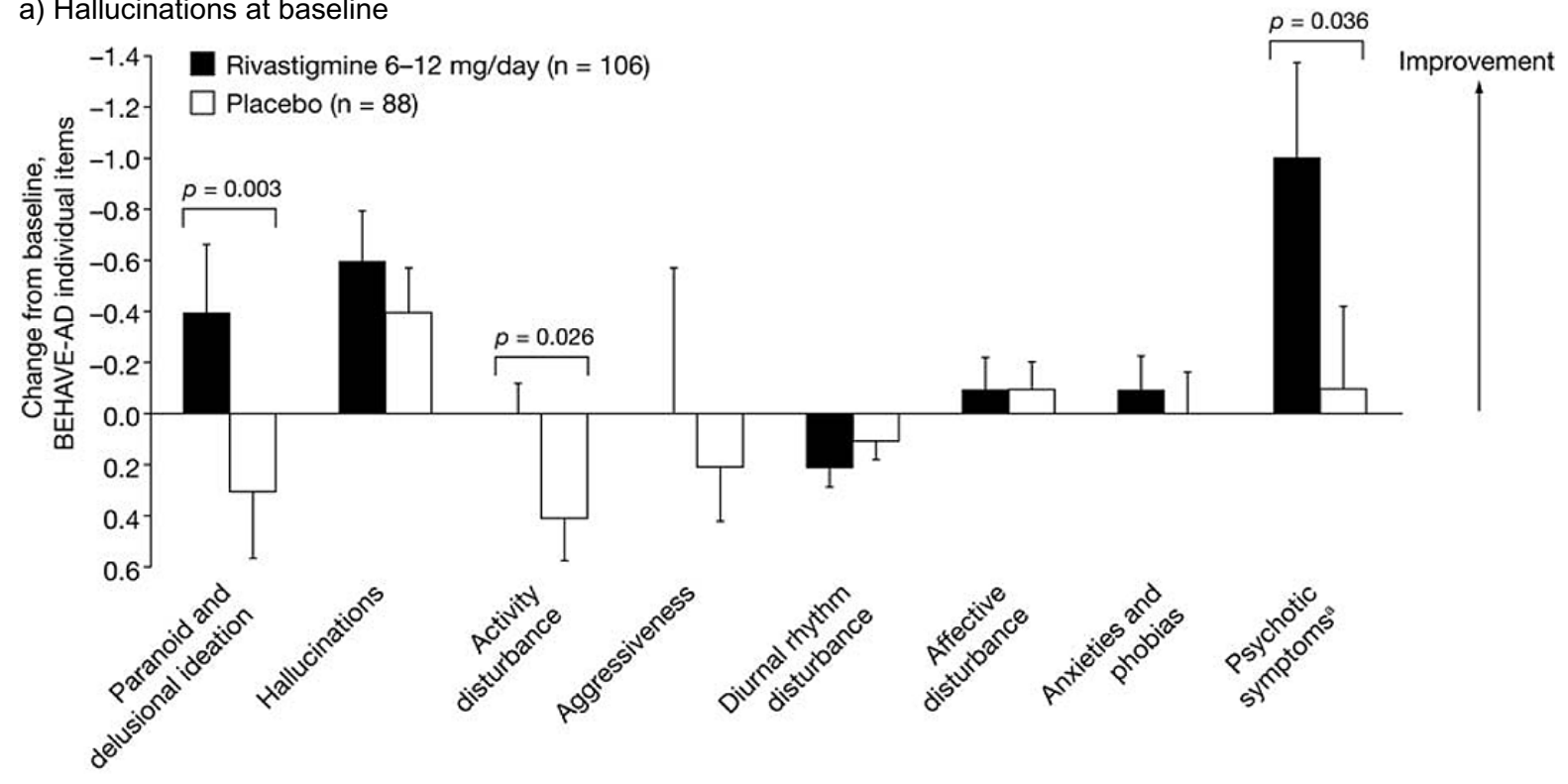

b) No hallucinations at baseline

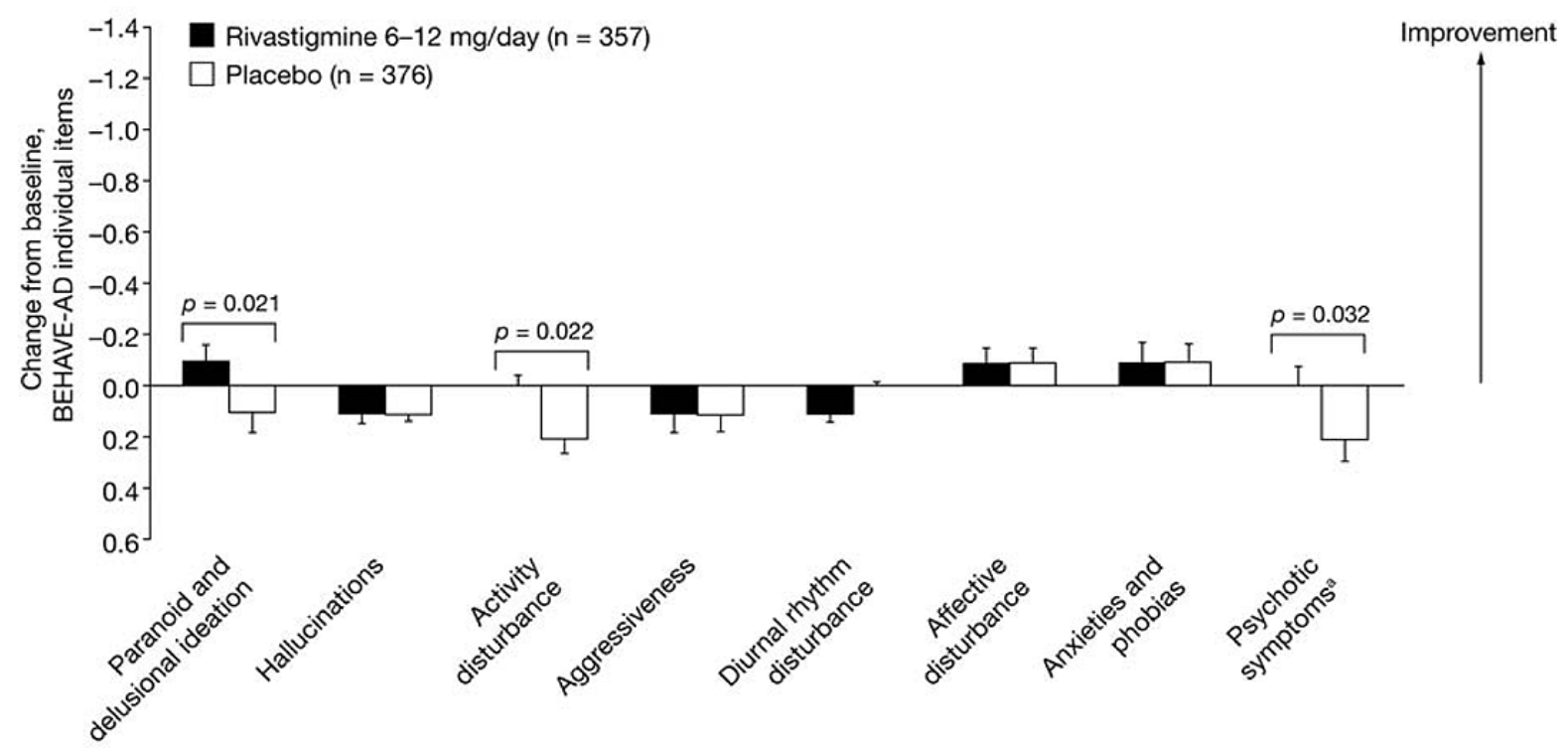

ITT analysis; $p$-value based on Van Elteren test; ahallucinations or delusions. Error bars indicate SEM.

Fig. 4. BEHAVE-AD scores at 6 months in AD patients a) with and b) without hallucinations at baseline receiving either rivastigmine 6-12 mg/day or placebo (SEM, standard error of the mean).

was carried out in this analysis. However, to evaluate what the impact of multiple comparisons on the results of this analysis would be if this analysis was not solely exploratory, a conservative Bonferroni method was applied to correct alpha, the level of significance for multiple comparisons. Using this method, the adjusted level of significance, corrected to account for the multiple comparisons of rivastigmine versus place- bo reported here, was $0.000962(<0.001)$. If this corrected level of significance were to be applied to the data reported here, the outcome (in terms of statistical significance) would remain unchanged for the results of the ADAS-cog; however, the results of the CIBICplus, PDS and MMSE would be significantly different between rivastigmine and placebo in the hallucinator group only, for all three study populations. The 
Table 3

Most frequently reported adverse events

\begin{tabular}{|c|c|c|c|c|}
\hline \multirow[b]{2}{*}{ Adverse event ${ }^{a}$} & \multicolumn{2}{|c|}{ Hallucinators $(\%)$} & \multicolumn{2}{|c|}{ Non-hallucinators $(\%)$} \\
\hline & $\begin{array}{c}\text { Rivastigmine } \\
(n=106)\end{array}$ & $\begin{array}{c}\text { Placebo } \\
(n=88)\end{array}$ & $\begin{array}{c}\text { Rivastigmine } \\
(n=356)\end{array}$ & $\begin{array}{c}\text { Placebo } \\
(n=376)\end{array}$ \\
\hline At least one adverse event & 90 & 82 & $93^{*}$ & 78 \\
\hline Nausea & $51 *$ & 8 & $52 *$ & 12 \\
\hline Vomiting & $33 *$ & 5 & $33^{*}$ & 5 \\
\hline Dizziness & $28 *$ & 10 & $22 *$ & 11 \\
\hline Anorexia & $25 *$ & 2 & $16^{*}$ & 3 \\
\hline Diarrhea & $24 *$ & 10 & $20 *$ & 13 \\
\hline Headache & 21 & 14 & $19^{*}$ & 12 \\
\hline Fatigue & 13 & 6 & $13^{*}$ & 4 \\
\hline Abdominal pain & 12 & 6 & $12 *$ & 6 \\
\hline Agitation & 11 & 18 & 7 & 5 \\
\hline Insomnia & 11 & 10 & 10 & 7 \\
\hline Accidental trauma & 10 & 6 & 7 & 9 \\
\hline Hallucination & 10 & 8 & 3 & 2 \\
\hline Confusion & 10 & 11 & 8 & 5 \\
\hline Asthenia & $9 *$ & 0 & 7 & 4 \\
\hline Depression & 8 & 7 & 6 & 5 \\
\hline Malaise & 8 & 3 & $6^{*}$ & $<1$ \\
\hline Coughing & 7 & 1 & $2 *$ & 5 \\
\hline Nervousness & 7 & 1 & 5 & 4 \\
\hline Upper respiratory tract infection & 6 & 7 & 8 & 9 \\
\hline Rhinitis & 6 & 1 & 4 & 4 \\
\hline Dyspepsia & 6 & 3 & $8^{*}$ & 3 \\
\hline Anxiety & 6 & 2 & 6 & 3 \\
\hline Hypertension & 6 & 1 & 3 & 2 \\
\hline Urinary tract infection & 5 & 5 & 8 & 6 \\
\hline Constipation & 5 & 3 & 4 & 4 \\
\hline Vertigo & 5 & 2 & 3 & 2 \\
\hline Ataxia & 5 & 1 & 1 & 1 \\
\hline Weight decrease & 5 & 0 & 2 & $<1$ \\
\hline Overdose & 5 & 7 & 4 & 6 \\
\hline Abnormal gait & 4 & 5 & $2 *$ & 0 \\
\hline Back pain & $<1$ & 6 & 4 & 4 \\
\hline Fever & $<1$ & 5 & $<1$ & $<1$ \\
\hline Increased sweating & 2 & 1 & $6^{*}$ & 2 \\
\hline Tremor & 4 & 1 & $5^{*}$ & 2 \\
\hline Flatulence & 3 & 2 & $5^{*}$ & 1 \\
\hline Pain & 4 & 2 & 3 & 5 \\
\hline
\end{tabular}

${ }^{a}$ Only those adverse events occurring in at least $5 \%$ of patients in any group are reported;

$* p<0.05$ versus placebo, Fisher's Exact Test.

results of the GDS would be significantly different between rivastigmine and placebo in the ITT population for the hallucinator group only, and the results of the BEHAVE-AD (total score and individual item scores) would no longer be significantly different between rivastigmine and placebo in hallucinators.

\section{DISCUSSION}

The aim of this pooled analysis was to compare the treatment effects of rivastigmine versus placebo in subpopulations of mild-to-moderate $\mathrm{AD}$ patients with and without hallucinations at baseline. A greater place- bo decline was observed in hallucinators compared with non-hallucinators. The presence of hallucinations was found to be associated with significantly greater rivastigmine versus placebo treatment differences on most efficacy outcomes in the primary analysis.

Rivastigmine treatment led to slight improvements or stabilization on measures of cognition and functioning in both hallucinators and non-hallucinators, but the increased placebo decline in patients with hallucinations at baseline resulted in significantly greater treatment differences on the ADAS-cog and PDS. On measures of global performance (CIBIC-plus and GDS), more improvement relative to placebo was seen in the hallucination group receiving rivastigmine. 
Hallucinations in AD have previously been associated with more rapid cognitive and functional decline [5], increased risk of mortality [28], and greater cortical cholinergic deficits [29-31]. In this study, while both hallucinators and non-hallucinators entered the study at a similar stage of AD as assessed by the MMSE and GDS, other outcome measures showed a trend towards greater deficits at baseline in hallucinators (Table 1). Cognition, as measured by the ADAS-cog, was more impaired at baseline in hallucinators compared with non-hallucinators (26.7 versus 21.7 points), as was the ability to perform activities of daily living, as assessed by the PDS (44.1 points versus 56.5 points for hallucinators and non-hallucinators respectively).

Analyses suggest that rivastigmine provides greater benefit in groups of patients experiencing a more aggressive course of dementia [29,30,32]. As in this study, larger drug placebo differences are observed in groups with greater decline. Although there is no standard definition for what constitutes an aggressive course of disease, the presence of hallucinations in a patient with dementia may predict a more rapid course of disease progression [32].

The cholinergic brainstem reticular formation system relays its influence on cortical function mainly through thalamic nuclei [33]. This cholinergic pathway is damaged in dementias associated with Lewy body pathology but is intact in most AD patients, except putatively those with hallucinations [34-36]. The hypothesis that brainstem cholinergic neuronal pathways are damaged in AD associated with hallucinations is supported by evidence of dysfunction of brainstem autonomic nuclei, with reduced vagally-mediated effects of rivastigmine on heart rate in hallucinators that were not observed in non-hallucinators. Rivastigmine has demonstrated larger treatment differences versus placebo in DLB and PDD patients with visual hallucinations, possibly due to the greater cholinergic neuronal deficits in areas of the brain associated with the pathophysiology of hallucinations $[11,12,37]$.

The findings of this retrospective pooled analysis should be interpreted with caution. Some of these patients, diagnosed in the 1980s and 90s as suffering from $\mathrm{AD}$ with hallucinations, may have fulfilled recently introduced criteria for the diagnosis of DLB. As no correction for multiplicity was carried out in this analysis, the results and $p$-values reported here are intended for hypothesis generation only. The data are consistent with previous studies in PDD and DLB patients receiving rivastigmine, and are suggestive of a differential treatment effect in hallucinators and non-hallucinators.
The comparison-adjusted data are consistent with the unadjusted analyses in suggesting that the effect of rivastigmine treatment is greater in patients with hallucinations.

In conclusion, statistically significant rivastigmine versus placebo treatment differences were seen in both hallucinators and non-hallucinators on all outcome measures reported in the pooled analyses of two trials of oral rivastigmine. The differences on cognitive, functional, global, and behavioral measures tended to be greater in patients with hallucinations at baseline compared with those without. There was greater decline in patients with hallucinations, suggesting that this subgroup has a more aggressive form of $\mathrm{AD}$.

\section{ACKNOWLEDGMENTS}

This study was supported by Novartis Pharma AG, Basel, Switzerland. ST, ND and RL are employees of Novartis. JC, ME and DA have provided consultation services to pharmaceutical companies that develop dementia drugs, including Novartis. Alpha-Plus Medical Communications Ltd assisted the authors in developing the first draft of this manuscript, based on a preliminary draft written by RL, and provided editorial support in the progress of subsequent drafts. This assistance was funded by Novartis Pharma AG. All authors reviewed and made substantial contributions to the content of this article, and approved it for submission.

Authors' disclosures available online (http://www.jalz.com/disclosures/view.php?id=251).

\section{REFERENCES}

[1] Ferri CP, Prince M, Brayne B, Brodaty H, Fratiglioni L, Ganguli M, Hall K, Hasegawa K, Hendrie H, Huang Y, Jorm A, Mathers C, Menezes PR, Rimmer E, Scazufca M (2005) Global prevalence of dementia: a Delphi consensus study. Lancet 366, 2112-2117.

[2] Davies P, Maloney AJ (1976) Selective loss of central cholinergic neurons in Alzheimer's disease. Lancet 2, 1403.

[3] Perry EK, Marshall E, Kerwin J, Smith CJ, Jabeen S, Cheng AV, Perry RH (1990) Evidence of a monoaminergiccholinergic imbalance related to visual hallucinations in Lewy body dementia. J Neurochem 55, 1454-1456.

[4] Robert P (2002) Understanding and managing behavioural symptoms in Alzheimer's disease and related dementias: focus on rivastigmine. Curr Med Res Opin 18, 156-171.

[5] Wilson RS, Gilley DW, Bennett DA, Beckett LA, Evans DA (2000) Hallucinations, delusions, and cognitive decline in Alzheimer's disease. J Neurol Neurosurg Psychiatry 69, 172177. 
[6] Ballard C, Bannister C, Graham C, Oyebode F, Wilcock G (1995) Associations of psychotic symptoms in dementia sufferers. Br J Psychiatry 167, 537-540.

[7] Street JS, Clark WS, Gannon KS, Cummings JL, Bymaster FP, Tamura RN, Mitan SJ, Kadam DL, Sanger TM, Feldman PD, Tollefson GD, Breier A (2000) Olanzapine treatment of psychotic and behavioral symptoms in patients with Alzheimer disease in nursing care facilities: a double-blind, randomized, placebo-controlled trial. The HGEU Study Group. Arch Gen Psychiatry 57, 968-976.

[8] Farber NB, Rubin EH, Newcomer JW, Kinscherf DA, Miller JP, Morris JC, Olney JW, McKeel, Jr DW (2000) Increased neocortical neurofibrillary tangle density in subjects with Alzheimer disease and psychosis. Arch Gen Psychiatry 57, 1165-1173.

[9] Lin SH, Yu CY, Pai MC (2006) The occipital white matter lesions in Alzheimer's disease patients with visual hallucinations. Clin Imaging 30, 388-393.

[10] Holroyd S, Shepherd ML, Downs, 3rd JH (2000) Occipital atrophy is associated with visual hallucinations in Alzheimer's disease. J Neuropsychiatry Clin Neurosci 12, 25-28.

[11] Burn D, Emre M, McKeith I, De Deyn PP, Aarsland D, Hsu C, Lane R (2006) Effects of rivastigmine in patients with and without visual hallucinations in dementia associated with Parkinson's disease. Mov Disord 21, 1899-1907.

[12] McKeith IG, Wesnes KA, Perry E, Ferrara R (2004) Hallucinations predict attentional improvements with rivastigmine in dementia with Lewy bodies. Dement Geriatr Cogn Disord 18, 94-100.

[13] Corey-Bloom J, Anand R, Veach J (1998) A randomized trial evaluating the efficacy and safety of ENA 713 (rivastigmine tartrate), a new acetylcholinesterase inhibitor, in patients with mild to moderately severe Alzheimer's disease. Int J Geriatr Psychopharmacol 1, 55-65.

[14] Rosler M, Anand R, Cicin-Sain A, Gauthier S, Agid Y, DalBianco P, Stahelin HB, Hartman R, Gharabawi M (1999) Efficacy and safety of rivastigmine in patients with Alzheimer's disease: international randomised controlled trial. BMJ 318, 633-638.

[15] American Psychiatric Association (1994) Diagnostic and Statistical Manual of Mental Disorders (DSM-IV), 4th ed.

[16] McKhann G, Drachman D, Folstein M, Katzman R, Price D, Stadlan EM (1984) Clinical diagnosis of Alzheimer disease: Report of the NINCDS-ADRDA Work Group under the auspices of Department of Health and Human Services Task Force on Alzheimer's disease. Neurology 34, 939-944.

[17] Anand R, Messina J, Hartman R (2000) Dose-response effect of rivastigmine in the treatment of Alzheimer's disease. Int $J$ Geriatr Psychopharmacol 2, 68-72.

[18] Schneider LS, Anand R, Farlow MR (1998) Systematic review of the efficacy of rivastigmine for patients with Alzheimer's disease. Int J Geriatr Psychopharmacol 1, S26-S34.

[19] Feldman H, Lane R (2007) Rivastigmine: a placebo-controlled trial of BID and TID regimens in patients with Alzheimer's disease. J Neurol Neurosurg Psychiatry 78, 1056-63.

[20] Rosen WG, Mohs RC, Davis KL (1984) A new rating scale for Alzheimer's disease. Am J Psychiatry 141, 1356-64.
[21] Reisberg B, Ferris SH (1994) CIBIC-Plus Interview Guide(c). Sandoz Pharmaceuticals Corporation: East Hanover, NJ, USA.

[22] DeJong R, Osterlund OW, Roy GW (1989) Measurement of quality-of-life changes in patients with Alzheimer's disease. Clin Ther 11, 545-54.

[23] Reisberg B, Ferris SH, de Leon MJ, Crook T (1982) The Global Deterioration Scale for assessment of primary degenerative dementia. Am J Psychiatry 139, 1136-1139.

[24] Folstein MF, Folstein SE, McHugh PR (1975) "Mini-mental state". A practical method for grading the cognitive state of patients for the clinician. J Psychiatr Res 12, 189-198.

[25] Reisberg B, Borenstein J, Salob SP, Ferris SH, Franssen E, Georgotas A (1987) Behavioral symptoms in Alzheimer's disease: phenomenology and treatment. J Clin Psychiat 48 (Suppl 5), 9-15.

[26] Cummings JL, Schneider E, Tariot PN, Graham SM (2006) Behavioral effects of memantine in Alzheimer disease patients receiving donepezil treatment. Neurology 67, 57-63.

[27] Gauthier S, Wirth Y, Mobius HJ (2005) Effects of memantine on behavioural symptoms in Alzheimer's disease patients: an analysis of the Neuropsychiatric Inventory (NPI) data of two randomised, controlled studies. Int J Geriatr Psychiatry 20, 459-464.

[28] Wilson RS, Krueger KR, Kamenetsky JM, Tang Y, Gilley DW, Bennett DA, Evans DA (2005) Hallucinations and mortality in Alzheimer disease. Am J Geriatr Psychiatry 13, 984-90.

[29] Cummings JL, Gorman DG, Shapira J (1993) Physostigmine ameliorates the delusions of Alzheimer's disease. Biol Psychiatry 33, 536-541.

[30] Kotrla KJ, Chacko RC, Harper RG, Jhingran S, Doody R (1995) SPECT findings on psychosis in Alzheimer's disease. Am J Psychiatry 152, 1470-1475.

[31] Levy ML, Cummings JL, Kahn-Rose R (1999) Neuropsychiatric symptoms and cholinergic therapy for Alzheimer's disease. Gerontology 45(Suppl 1), 15-22.

[32] Gauthier S, Vellas B, Farlow M, Burn D (2006) Aggressive course of disease in dementia. Alzheimers Dement 2, 210-217.

[33] McKinney M, Jacksonville MC (2005) Brain cholinergic vulnerability: relevance to behavior and disease. Biochem Pharmacol 70, 1115-1124.

[34] Sassin I, Schultz C, Thal DR, Rub U, Arai K, Braak E, Braak H (2000) Evolution of Alzheimer's disease-related cytoskeletal changes in the basal nucleus of Meynert. Acta Neuropathol 100, 259-269.

[35] Tiraboschi P, Hansen LA, Alford M, Sabbagh MN, Schoos B, Masliah E, Thal LJ, Corey-Bloom J (2000) Cholinergic dysfunction in diseases with Lewy bodies. Neurology 54, 407411.

[36] Bohnen NI, Kaufer DI, Ivanco LS, Lopresti B, Koeppe RA, Davis JG, Mathis CA, Moore RY, DeKosky ST (2003) Cortical cholinergic function is more severely affected in parkinsonian dementia than in Alzheimer disease: an in vivo positron emission tomographic study. Arch Neurol 60, 1745-1748.

[37] Ballard C, Lane R, Barone P, Ferrara R, Tekin S (2006) Cardiac safety of rivastigmine in Lewy body and Parkinson's disease dementias. Int J Clin Pract 60, 639-645. 